\title{
Temporal trends without seasonal effects on gestational diabetes incidence relate to reductions in indices of insulin secretion: the Cambridge Baby Growth Study
}

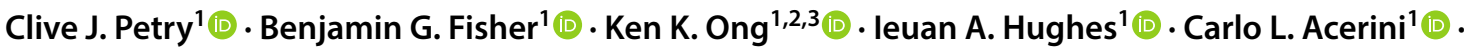 \\ David B. Dunger ${ }^{1,3}$
}

Received: 3 April 2019 / Accepted: 26 April 2019 / Published online: 13 May 2019

(c) The Author(s) 2019

\begin{abstract}
Aims The incidence of gestational diabetes has been reported to have risen over the first decade of this century. Some studies have also found it to vary with seasons of the year. We investigated temporal and seasonal trends on gestational diabetes incidence in a single-centre cohort study from Cambridge, UK, and attempted to explain trends using associated risk factors. Methods Using a cosinor model, we tested both temporal and seasonal trends in gestational diabetes incidence in 1074 women recruited to the Cambridge Baby Growth Study in 2001-2009 who underwent oral glucose tolerance tests around week 28 of pregnancy.

Results There was a temporal increase in gestational diabetes incidence over the course of recruitment to this study [0.014 $(0.005,0.022)$ proportional increase per year, $\left.p=2.1 \times 10^{-3}\right]$, but no seasonal effect $(p=0.7)$. HOMA B $[-0.015(-0.025$, $-0.005)$ per year, $\left.p=3.0 \times 10^{-3}\right]$ and the insulin disposition index $\left[-0.036(-0.060,-0.013)\right.$ per year, $\left.p=3.0 \times 10^{-3}\right]$, unlike HOMA S, showed negative temporal trends. Risk factor analyses showed a concomitant temporal slight increase in the index of multiple deprivation $\left[0.191(0.138,0.257)\right.$ units per year, $\left.p=4.6 \times 10^{-10}\right]$. This index was positively associated with HOMA B $\left(p=6.1 \times 10^{-5}\right)$ but not directly with gestational diabetes $(p=0.6)$, HOMA S $(p=0.2)$ or the insulin disposition index $(p=0.4)$.

Conclusions In this cohort, there were temporal, but not seasonal, increases in gestational diabetes incidence between the years 2001 and 2009, which appeared to be related more to reductions in insulin secretion than sensitivity. Possible mediators of this link include confounding factors related to deprivation.
\end{abstract}

Keywords Insulin sensitivity $\cdot$ Pregnancy $\cdot$ Deprivation $\cdot$ Cosinor

Managed by Antonio Secchi.

Clive J. Petry

cjp1002@cam.ac.uk

1 Department of Paediatrics, University of Cambridge, Box 116, Cambridge Biomedical Campus, Hills Road, Cambridge CB2 0QQ, UK

2 Medical Research Council Epidemiology Unit, University of Cambridge, Cambridge CB2 0QQ, UK

3 The Institute of Metabolic Science, University of Cambridge, Cambridge CB2 0QQ, UK

\section{Introduction}

Gestational diabetes (GDM) is traditionally defined as carbohydrate intolerance with its onset or the first recognition in pregnancy [1], although more recent definitions explicitly exclude pre-existing type 2 diabetes [2]. It is one of the most common adverse conditions of pregnancy. Its incidence has generally been reported to be rising in most populations, usually in line with the increasing prevalence of maternal obesity [3]. Other major risk factors for GDM include having a previous history of it or having previously given birth to a macrosomic baby, a family history of GDM and/or type 2 diabetes, increased maternal age, increased gestational weight gain, genetics, multiparity and ethnic factors [4], not all of which can explain the tempo of the rising incidence. As GDM increases the risk of short- and 
long-term adverse complications for both the mother and her unborn child (including macrosomia, pre-eclampsia, childhood obesity and the metabolic syndrome in the mother [5]) and may contribute to the diabetes pandemic [6], a thorough understanding of its pathogenesis is essential.

With the apparent worldwide rise in the prevalence of GDM [7], paralleling the increasing prevalence of female obesity [8] of note is the temporal and potentially mechanistic links that obesity has with global warming [9]. Therefore, another factor that could explain at least part of the increased incidence of GDM is exposure to raise or rising ambient temperatures in certain populations [10-12]. Following this, some studies have reported seasonal variations in the incidence of GDM [13-17], although this has not been observed in all populations or climates $[18,19]$. In this study we investigated whether there were temporal and seasonal trends in GDM incidence in our single-centre population from Cambridge, UK, which recruited pregnant women between 2001 and 2009. We then investigated what may have mediated any trends. Although relating to a decade ago, this seemed reasonable given that GDM was already becoming more prevalent by this time in several different populations [20-25].

\section{Materials and methods}

\section{Cambridge Baby Growth Study}

The prospective, longitudinal Cambridge Baby Growth Study (CBGS) was established as an observational cohort initially covering pregnancy, birth and infancy [26]. 2229 mothers, all over 16 years of age, were recruited when attending early pregnancy ultrasound clinics at the Rosie Maternity Hospital, Cambridge, UK, between April 2001 and March 2009. A total of 571 of these mothers withdrew prior to the birth of their infant. Most of the clinical characteristics of the study participants were collected either during nurse-led interviews or by questionnaire with the exception of offspring birth weight, gestational age and date of birth, which were compiled from hospital notes. In this cohort, $95.3 \%$ of the offspring were white, $1.7 \%$ were Asian, $1.3 \%$ were black (African or Caribbean) and $1.7 \%$ were other ethnicities (mainly mixed race), reflective of the population served by the Rosie Maternity Hospital.

\section{Ethics}

The Cambridge Baby Growth Study was approved by the Cambridge Local Research Ethics Committee, Addenbrooke's Hospital, Cambridge, UK (00/325). All procedures followed were in accordance with the institutional guidelines. Written informed consent was obtained from all the study participants.

\section{Oral glucose tolerance test and gestational diabetes diagnostic criteria}

At a median (inter-quartile range) of 28.4 (28.1-28.7) weeks gestation, 1074 of the CBGS mothers underwent a 75-g oral glucose tolerance test (OGTT) after fasting overnight [27]. Venous blood was collected just prior to and $60 \mathrm{~min}$ after the glucose load was administered for the measurement of plasma glucose, insulin and c-peptide concentrations. 120 min plasma glucose concentrations were only measured from May 2007 onwards so were not used in this study to define GDM (only 7\% of UK women with GDM receive a diagnosis based solely on the 120-min measurement in any case [28]). The International Association of Diabetes in Pregnancy Study Groups (IADPSG) thresholds for 0 and $60 \mathrm{~min}$. OGTT glucose concentrations (i.e. $\geq 5.1$ and $10.0 \mathrm{mmol} / \mathrm{L}$, respectively [29]) were used to define the presence of GDM.

\section{Assays}

All biochemical kit-based assays were run according to the manufacturer's instructions. Glucose concentrations were measured using a routine glucose oxidase-based method. OGTT plasma insulin concentrations were measured by ELISA (Dako UK Ltd., Ely, Cambs, UK). Intra-assay imprecision $(\mathrm{CV})$ was $4.3 \%$ at $14 \mathrm{mU} / \mathrm{L}(82 \mathrm{pmol} / \mathrm{L}), 3.0 \%$ at $67 \mathrm{mU} / \mathrm{L}(402 \mathrm{pmol} / \mathrm{L})$ and $5.7 \%$ at $151 \mathrm{mU} / \mathrm{L}$ (907 pmol/L). Equivalent inter-assay imprecision was 4.3, 5.1 and 5.4\%, respectively. C-peptide concentrations were also measured by ELISA (DSL Labs., London, UK). Intra-assay imprecision was $2.8 \%$ at $1.3 \mathrm{ng} / \mathrm{mL}(0.43 \mathrm{nmol} / \mathrm{L})$ and at $4.4 \mathrm{ng} /$ $\mathrm{mL}(1.47 \mathrm{nmol} / \mathrm{L})$ and $3.2 \%$ at $8.4 \mathrm{ng} / \mathrm{mL}(2.80 \mathrm{nmol} / \mathrm{L})$. Equivalent inter-assay imprecision was $15.7,7.8$ and $10.3 \%$, respectively.

\section{Calculations}

The maternal body mass index (BMI) was calculated as the pre-pregnancy weight divided by the height squared. Insulin sensitivity and pancreatic $\beta$-cell function were estimated using the homeostasis model assessment (HOMA S and B, respectively), calculated using the week 28 fasting circulating glucose and insulin (or c-peptide) concentrations and the online HOMA2 calculator (available at https://www.dtu. ox.ac.uk/homacalculator/) [30]. For this study, HOMA values were calculated using both insulin and c-peptide concentrations separately, and where mentioned in this manuscript refer to insulin-derived values unless stated otherwise. Insulin secretion (for a given insulin sensitivity) was assessed 
in terms of the insulin disposition index, calculated as the change in insulin concentrations over the first hour of the OGTT divided by the change in glucose concentrations, all divided by the reciprocal of the fasting insulin concentration. An equivalent disposition index was also calculated using plasma c-peptide concentrations. The index of multiple deprivation was derived and imputed from the postcode of the participants' home addresses as described [31].

\section{Statistical analysis}

The present analysis was restricted to those 1074 pregnancies where the women underwent OGTTs (thereby, excluding women with pre-existing type 1 diabetes) with 0 and 60 min plasma glucose concentrations available to us. Data were logarithmically transformed prior to analyses if their distributions were positively skewed. Temporal trends, adjusted for seasonal trends, were tested for using the cosinor regression model in $\mathrm{R}$ (version 3.5.2; The R Project for Statistical Computing, Vienna, Austria) deploying the package cosinor (version 1.1, available at http://github.com/ sachsmc/cosinor) which assumes a sinusoidal seasonal pattern over a longitudinal period, in this case OGTT season and year of analysis, respectively. The season was based on the month the OGTT was performed using Northern Meteorological seasons: winter (December-February), spring (March-May), summer (June-August) and autumn (fall) (September-November). Further analysis was performed by standard logistic (for binary variables) or linear (for continuous variables whose model residuals using untransformed or transformed data were normally distributed) regression. Nonparametric regression (to fit linear regression models that included the index of multiple deprivation due to the lack of normal distribution of the residuals in standard linear regression models) was performed by means of the Siegel repeated medians procedure (deploying the $\mathrm{R}$ package mblm, version 0.12.1, available at https://cran.r-project.org/ web/packages/mblm/index.html). Categorical analysis was performed using the $\chi^{2}$ test. Unless stated, all the other statistical analyses were performed using Stata (version 13.1; Stata Corp., from Timberlake Consultants Ltd., Richmond, Surrey, UK). Statistical significance was assumed at $p<0.05$ throughout.

GDM risk factors assessed as above for association with year of testing included: BMI, pregnancy weight gain, maternal age, offspring birth weight, parity and index of multiple deprivation (as continuous variables), and offspring sex and multifetal pregnancy (as categorical/binary data).

\section{Results}

\section{Characteristics of the study population}

With the exception of a slight increase in parity and a reduced proportion of smokers, those women who were included in the analysis were representative of the Cambridge Baby Growth Study as a whole (Table 1). Variables that were not detectably different included GDM prevalence, fasting glucose and insulin concentrations, pre-pregnancy BMI and maternal age.

\section{Temporal Trends of GDM Incidence and OGTT Glucose Concentrations}

The overall prevalence of GDM in this population was $10.1 \%$ (108/1071). Cosinor analysis showed a significant temporal effect associated with the year of analysis [0.014

Table 1 Characteristics of those Cambridge Baby Growth Study participants who were included in the current analysis and those that were not

\begin{tabular}{llll}
\hline Characteristic & Included & Not included & $p$ value \\
\hline Mother's age at the birth of her baby (years) & $33.4(33.1,33.6)(n=901)$ & $33.8(33.4,34.2)(n=434)$ & $228 / 267 / 93 / 29 / 2 / 3$ \\
Parity $(n$, of increasing parity) & $489 / 373 / 122 / 32 / 6 / 3$ & 1 yes, 6 no & 0.06 \\
GDM & 110 yes, 967 no & $4.2(3.7,4.6)(n=6)$ & 0.5 \\
OGTT fasting glucose concentration (mmol/L) & $4.3(4.3,4.4)(n=1073)$ & $46.4(41.3,52.1)(n=83)$ & 0.5 \\
OGTT fasting insulin concentration (pmol/L) & $45.5(44.0,47.0)(n=1053)$ & $23.3(22.9,23.7)(n=357)$ & $0.0(8.7,9.2)(n=585)$ \\
Pre-pregnancy BMI $\left(\mathrm{kg} / \mathrm{m}^{2}\right)$ & $23.4(23.2,23.7)(n=830)$ & $33 / 52 / 134$ & 0.6 \\
Index of multiple deprivation & $8.9(8.7,9.1)(n=1068)$ & $320 / 307$ & 0.9 \\
Maternal highest qualification (category 3/4/5) & $80 / 133 / 358$ & $3.458(3.412,3.504)(n=357)$ \\
Sex of baby $(n$ males/females) & $535 / 492$ & $39.7(39.6,39.8)(n=628)$ \\
Baby's birth weight $(\mathrm{kg})^{\mathrm{a}}$ & $3.483(3.454,3.514)(n=830)$ & $46 / 582$ & 0.4 \\
Gestational age at birth of baby (weeks) & $39.8(39.7,39.9)(n=1029)$ & 0.1 \\
Reported smoking during pregnancy $(n$ yes/no) & $40 / 987$ & $2.0 \times 10^{-3}$ \\
\hline
\end{tabular}

${ }^{a}$ Adjusted for maternal pre-pregnancy BMI, gestational age at birth, sex of baby and parity. Data are either number of participants or mean (95\% confidence interval) 
$(0.005,0.022)$ proportional increase per year, $\left.p=2.1 \times 10^{-3}\right]$, with a trend for the incidence of GDM increasing through the years 2001-2009 (Fig. 1). This finding was confirmed by logistic regression [odds ratio $(\mathrm{OR}) 1.2(1.1,1.3)$ per year, $p=2.8 \times 10^{-3}, n=1049$ ], even when the small numbers of participants collected in 2009 were excluded from the analysis [OR $1.2(1.1,1.3)$ per year, $p=3.3 \times 10^{-3}, n=1040$ ]. There was, however, no significant association of GDM with seasonality [amplitude: $9.8(-14.7,34.3), p=0.4$; acrophase $-0.6(-3.0,1.9), p=0.7]$ (Fig. 1). Cosinor analysis revealed no significant association of the OGTT fasting glucose concentration with the year $[0.009(-0.007,0.025) \mathrm{mmol} / \mathrm{L}$ per year, $p=0.3$ ] or with seasonality [amplitude: $21.3(-24.1$, $66.6), p=0.4$; acrophase $-0.4(-2.4,1.6), p=0.7]$. Conversely there was a temporal trend with the OGTT 60-min glucose concentrations $[0.117(0.067,0.166) \mathrm{mmol} / \mathrm{L}$ per year, $p<1 \times 10^{-4}$ ] (Fig. 2), a finding which was confirmed
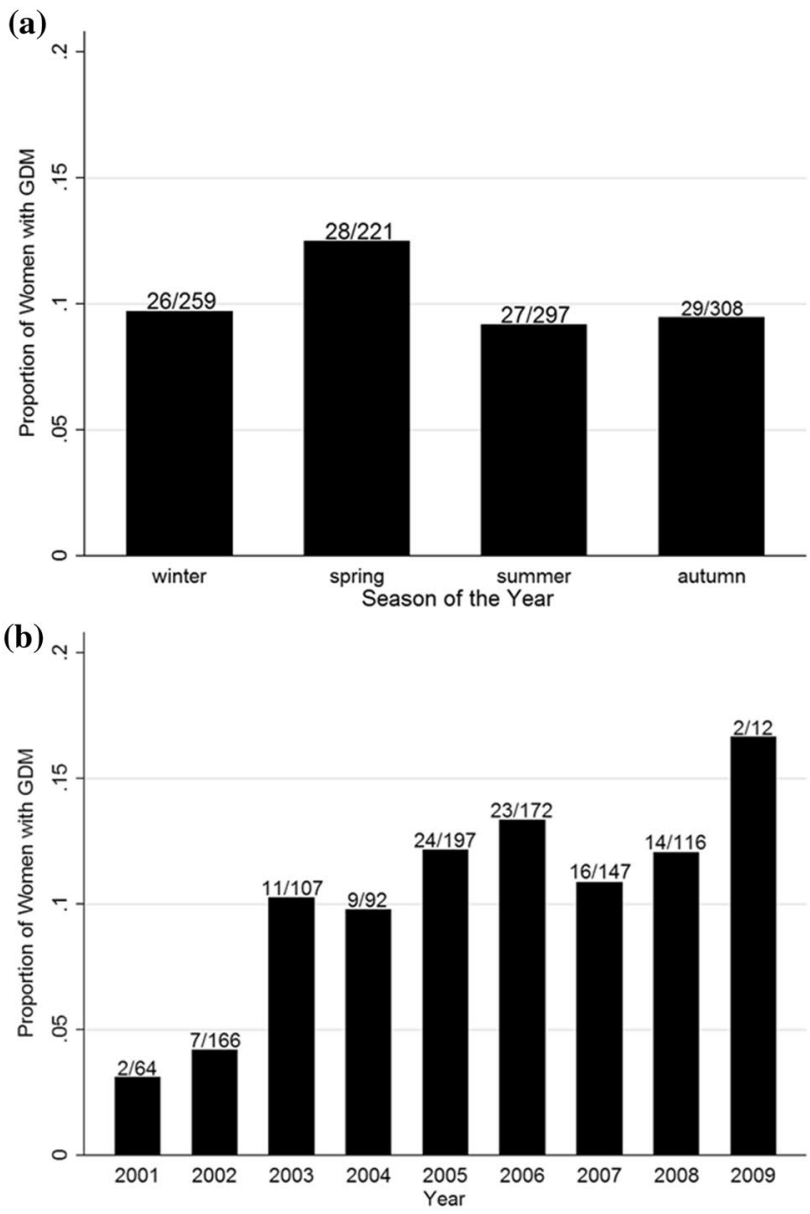

Fig. 1 a Proportion of women with GDM by the season of the year, following an OGTT around week 28 of pregnancy, adjusted for year of analysis. Data are means (95\% confidence intervals). Analysing this data categorically (taking no account of the recurring order of the seasons) showed no association between GDM and season of the year $\left(\chi^{2}=2.2, p=0.5\right)$. b Proportion of women with GDM by the year of testing, following an OGTT around week 28 of pregnancy by linear regression $\left(\beta^{\prime}=0.147, p=1.3 \times 10^{-6}, n=1071\right)$, without a seasonal effect [amplitude: $53.7(-84.1,191.5)$, $p=0.4$; acrophase $-0.9(-3.4,1.7), p=0.5]$.

\section{Temporal trends of indices of insulin sensitivity and secretion}

Insulin-derived HOMA $\mathrm{S}$ was positively associated with the year of testing $[0.016(0.001,0.032)$ per year, $p=0.04]$ but not with the season of testing [amplitude: $21.7(-20.8$, $64.1), p=0.3$; acrophase $1.3(-0.7,3.4), p=0.2]$, a finding which was confirmed by linear regression $\left(\beta^{\prime}=0.080\right.$, $\left.p=9.1 \times 10^{-3}, n=1049\right)$. This association was still evident if adjusting for GDM ( $\left.p=3.4 \times 10^{-4}, n=1049\right)$ or by only including women who were classified as not having GDM $\left(p=1.2 \times 10^{-3} ; n=947\right)$. However, the association disappeared if it were tested using c-peptide-derived HOMA S, in a smaller number of women $\left(\beta^{\prime}=0.014, p=0.7, n=929\right)$.

Whilst still having a lack of association with seasonality [amplitude: $20.9(-6.4,48.2), p=0.1$; acrophase $0.6(-0.7$, $1.9), p=0.4$ ], in contrast to the findings with HOMA S, insulin-derived HOMA B was negatively associated with year of testing $\left[-0.015(-0.025,-0.005)\right.$ per year, $\left.p=3.0 \times 10^{-3}\right]$. This association was confirmed by linear regression $\left(\beta^{\prime}=-0.104, p=7.0 \times 10^{-4}, n=1049\right)$. Again, it was still evident if adjusting for $\operatorname{GDM}\left(p=1.2 \times 10^{-3}, n=1049\right)$ or if only including women without GDM $\left(p=1.2 \times 10^{-3}\right.$; $n=947)$. This association was still present if it were tested using c-peptide-derived HOMA B, in a smaller number of women $\left(\beta^{\prime}=-0.073, p=0.03, n=929\right)$.

Similar to the results for HOMA B, the insulin disposition index was negatively associated with year of testing $\left[-0.036(-0.060,-0.013)\right.$ per year, $\left.p=3.0 \times 10^{-3}\right]$, a finding which was confirmed by linear regression $\left(\beta^{\prime}=-0.091\right.$, $\left.p=3.8 \times 10^{-3}, n=1000\right)$. This association was still evident if adjusting for GDM $(p=0.03 ; n=1000)$ or if only including women without GDM $(p=0.03 ; n=905)$. The insulin disposition index was not, however, associated with season of testing [amplitude: $26.0(-38.5,90.5), p=0.4$; acrophase $1.1(-1.5,3.7), p=0.4]$. The c-peptide disposition index was also negatively associated with year of testing $\left(\beta^{\prime}=-0.074\right.$, $p=0.03, n=882$ ).

\section{Associations of the year of analysis with potential risk factors}

The year of analysis was not associated with either the maternal BMI $\left(\beta^{\prime}=0.006, p=0.9, n=827\right)$ or pregnancy weight gain $\left(\beta^{\prime}=-0.047, p=0.2, n=614\right)$. Neither was it associated with maternal age $\left(\beta^{\prime}=-0.016, p=0.6, n=898\right)$ or parity ( $\beta^{\prime}=0.007, p=0.8, n=1022$ ) in this population. There was no association between the year of analysis and the proportion of male babies $\left(\chi^{2}=4.9, p=0.8, n=1024\right)$, 
Fig. 2 Dot plot of OGTT 60-min glucose concentrations by the year of OGTT testing. The bars represent the limits of the inter-quartile range of the values represented by the dots

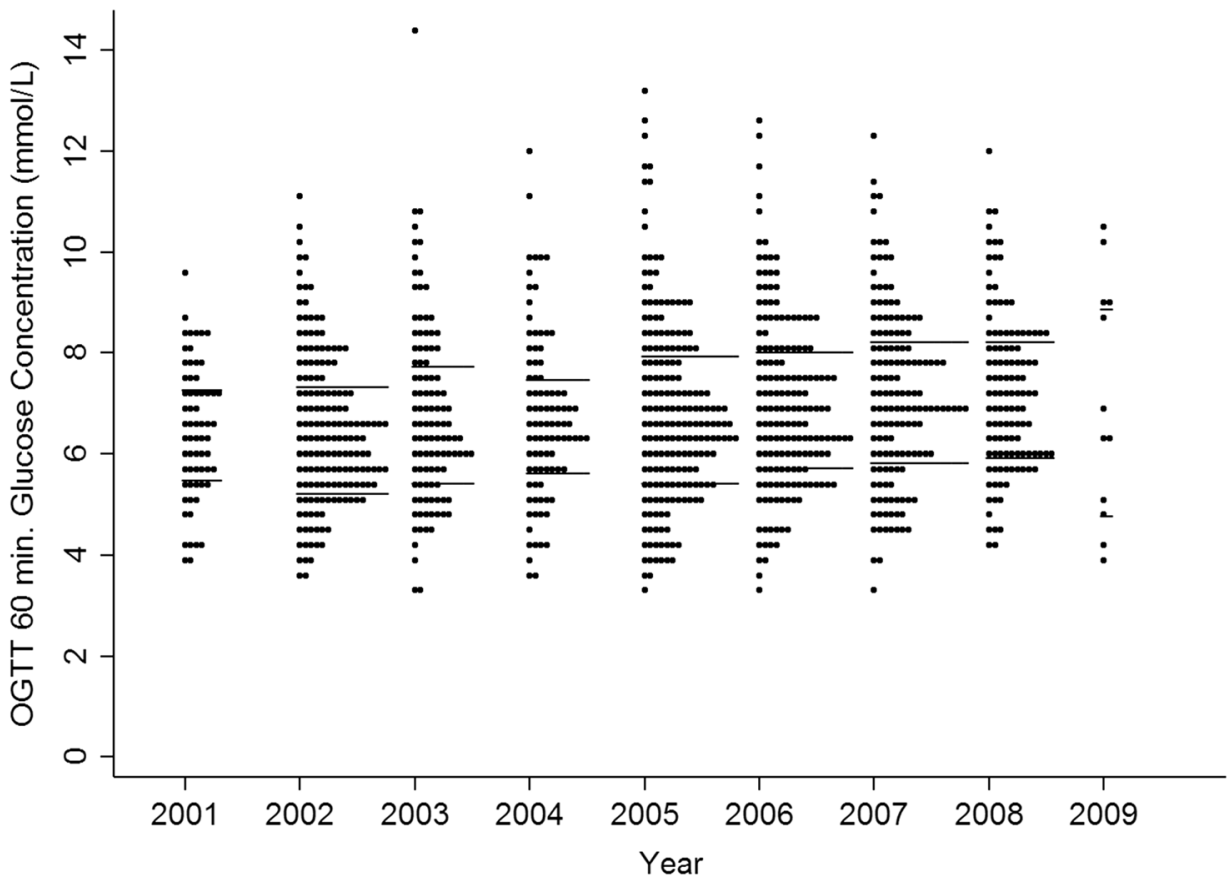

birth weight of the babies $\left(\beta^{\prime}=-0.023, p=0.4, n=824\right.$; adjusted for gestational age at birth, mother's BMI, parity and sex) or the odds of a pregnancy being multifetal [OR $1.0(0.9,1.2), p=0.9, n=1071]$. One potential confounder that year of analysis was modestly albeit highly significantly associated with, and the index of multiple deprivation [0.191 $(0.138,0.257)$ units per year, $\left.p=4.6 \times 10^{-10}, n=1068\right]$ (Fig. 3) was itself not directly associated with GDM [OR 1.0 $(1.0,1.1)$ per unit increase in the index, $p=0.6, n=1032]$. There was still a significant relationship between the index of multiple deprivation and the year in the reduced number of 955 women for whom HOMA modelling was available $\left[0.174(0.118,0.237)\right.$ units per year, $\left.p=5.2 \times 10^{-8}\right]$. In these women, the index of multiple deprivation was significantly positively associated with HOMA B [0.756 (0.376, 1.143) per unit, $p=6.1 \times 10^{-5}$; Fig. 3] but not with HOMA $\mathrm{S}[-0.394(-0.967,0.197)$ per unit, $p=0.2]$ or the insulin disposition index $[43.6(-53.8,150.8)$ per unit, $p=0.4]$.

\section{Discussion}

In this analysis, there was a strong trend for the incidence of GDM increasing as recruitment to the cohort progressed between 2001 and 2009. Trends over a similar period of time have also been observed in populations in Canada [21], the USA [22, 24], Israel [23] and Germany [25]. The worldwide increased incidence at that time appeared to be independent of ethnicity [20] despite this being a major factor associated with GDM risk [4]. In our population, the increased incidence of GDM was associated with reductions in HOMA
$\mathrm{B}$ and the insulin disposition index (rather than HOMA S). Whilst a temporal trend in these factors could relate to changes in the performance of the insulin and glucose assays that were used over time, we used the same assays for these analyses throughout this time period and the performance characteristics of the insulin assay matched those of similar assays available at the time [32]. Given that the performance of the glucose assay did not change over this time, the fact that the associations with HOMA B and the disposition index persisted if they were calculated using c-peptide rather than insulin concentrations suggests that these associations were physiological rather than assay related. The temporal increase in GDM incidence in our population therefore appeared to relate to reductions in insulin secretion rather than sensitivity (indeed insulin-derived HOMA S actually increased over the period of recruitment, albeit this association was not evident when c-peptide concentrations were used to calculate HOMA $S$ in a smaller number of women).

The temporal increase in GDM incidence was clearly environmentally mediated as its tempo was too fast for a genetic change. In investigating its potential causes and the reduced insulin secretion, we could not find parallel increases in BMI (itself strongly associated with GDM and partially driving the link between factors such as early age at menarche and increased GDM risk [33] as has previously been observed in our population [34]), pregnancy weight gain, maternal age or smoking. There was, however, a significant temporal trend of increasing deprivation in the study (in a population that was generally less-deprived than the national average [26]) although deprivation itself was not associated with GDM. There was a modest albeit highly 
Fig. 3 Dot plots of a the index of multiple deprivation by year of testing and b HOMA B values by the index of multiple deprivation. The lines of best fit (minimising the distance between the line and median values) were calculated using the Siegel repeated medians procedure
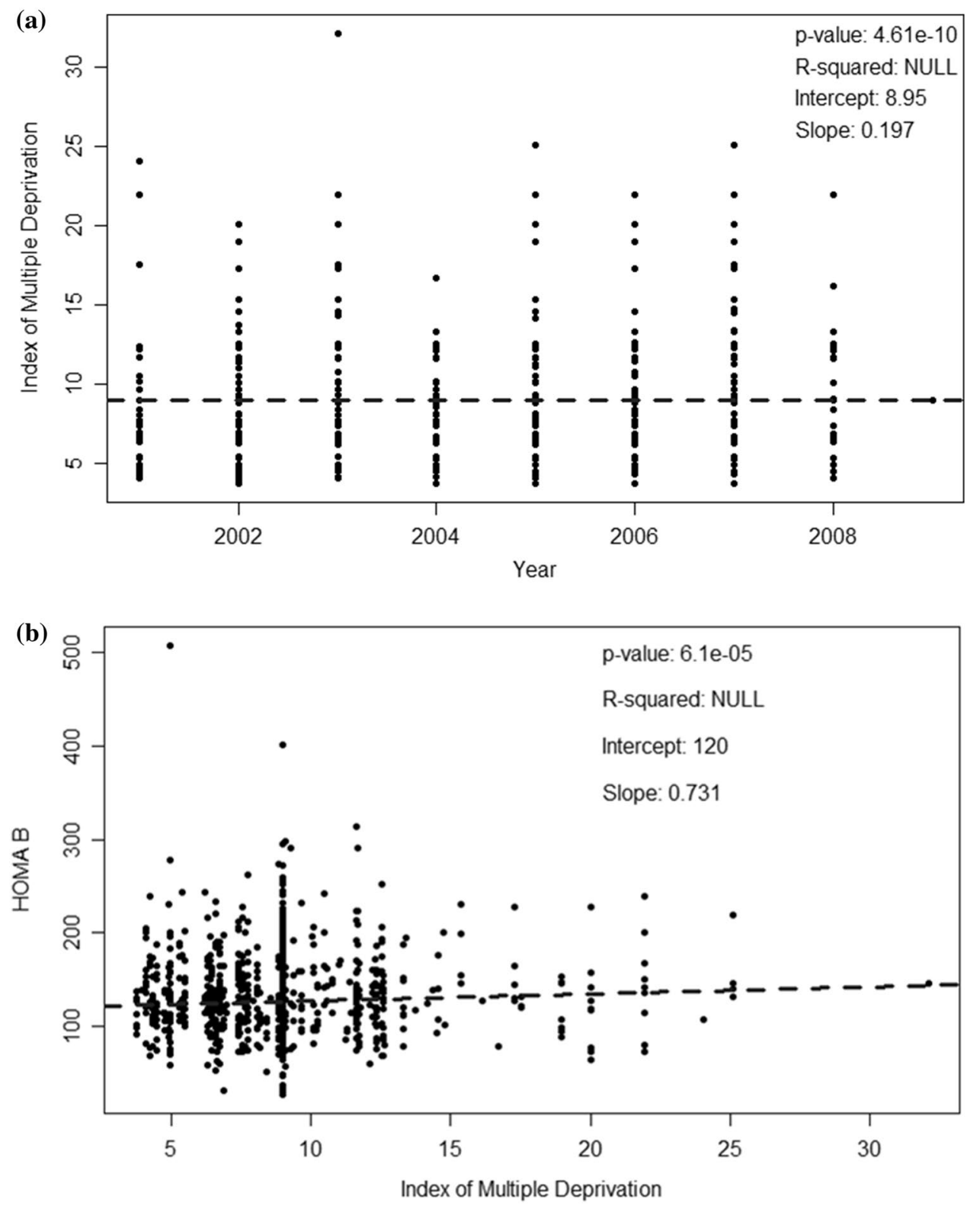

significant positive association between the index of multiple deprivation and insulin-derived HOMA B. This suggests that in the absence of direct associations with HOMA S or GDM risk, as the deprivation index went up pancreatic $\beta$-cell function might have had to increase slightly to maintain glucose homeostasis. Due to the lack of direct association between the deprivation index and GDM despite the temporal trend, it is possible, however, that as yet unidentified confounder(s) related to deprivation, such as factors connected with diet [35] and/or exercise [36], could have contributed to the temporal increase in GDM incidence. This would be consistent with studies where associations between GDM and deprivation, lower socioeconomic status or lower social class were reported [25, 37, 38] although such associations have not been found in every study [39]. An alternative explanation for the temporal trend in deprivation in the present study is that it may just reflect unintentional secular patterning in study recruitment or uptake.

Although we observed a temporal trend of increasing GDM incidence as the decade progressed in this analysis, we could not find a seasonal trend. This is despite seasonal trends in GDM previously have been observed in populations in Sweden [13], Australia [14, 15], Italy [16] and Greece [17]. However, of the populations tested before where no such trend was observed [18, 19], one of these was also in the UK [19] so our lack of seasonal trend may relate to climate or other environmental factors specific to the UK. GDM is known to be a heterogeneous disease [40], and the degree of that heterogeneity may be smaller when comparing two populations of the same nationality 
and ethnic mix. Alternatively, whilst at least one of the studies that found a seasonal trend used a very similar analysis technique to the one that we used [15], other studies used analysis of variance or categorical/ordinal analyses which did not account for the recurring nature of the seasons or adjust for longer-term temporal trends $[13,14$, $16,17]$ so differences from our results may relate to this.

The strengths of our prospective study include the fact that we used cosinor analysis to adjust linear temporal trends for separate potential seasonal effects, unlike some of the other studies in this area. In addition, we had third trimester OGTT data from all the study participants and so we were able to investigate whether temporal trends in GDM incidence were related to changes in insulin sensitivity or secretion, a level of detail that is rarely available in larger surveys. We calculated HOMAs using both insulin and c-peptide concentrations so that detectable temporal trends were less likely to have resulted from drift in assay performance. In addition to its strengths, the study does have a number of limitations, however. Firstly, this Cambridge cohort may not fully reflect the UK population as a whole particularly in relation to ethnic mix and smoking prevalence [25], although this means that there was probably less confounding due to ethnic effects and other factors related to the heterogeneity of GDM [40]. Secondly, although the study was fairly large given the level of detail that was collected, it was smaller than temporal studies of large surveys (e.g. [41]), and therefore, the proportion of women who develop GDM in our study may be a little unrepresentative of the whole population. This could partially account for the large magnitude of the increased GDM incidence over the course of the recruitment period in our study. This limitation is offset by the level of detail that was collected for each of the study participants, however, which allowed us to relate temporal changes in the prevalence of GDM to changes in insulin secretion, a finding which has not been attempted in larger studies. Thirdly, another limitation is that we did not record family histories of GDM and type 2 diabetes, major risk factors for GDM [4]. The final limitation of our study is that the insulin (and c-peptide) disposition index was not calculated using $30 \mathrm{~min}$. OGTT glucose and insulin concentrations like usual, but using 60 min concentrations instead. Although comparing OGTT results from our study with those more commonly from intravenous GTTs, its use has been deemed acceptable at least for the insulinogenic element of the disposition index [42].

In conclusion, we observed a temporal but not seasonal trend for an increasing incidence of GDM in Cambridge Baby Growth Study pregnancies from the years 2001-2009. This was associated with reductions in indices of insulin secretion rather than insulin sensitivity. Although we do not know what caused these changes, it does not appear to relate to changes in maternal obesity or age. Factors relating to deprivation offer potential explanations.

Acknowledgements The authors would like to thank all the families that took part in the Cambridge Baby Growth Study and acknowledge the crucial role played by the research nurses, especially Suzanne Smith, Ann-Marie Wardell and Karen Forbes, staff at the Addenbrooke's Wellcome Trust Clinical Research Facility and midwives at the Rosie Maternity Hospital in collecting data for this study. We would also like to thank Angie Watts, Karen Whitehead and Dianne Wingate for excellent laboratory assistance. This work was supported by the Medical Research Council (Grant Numbers G1001995, 7500001180); European Union Framework 5 (Grant Number QLK4-1999-01422); the Mothercare Charitable Foundation (Grant Number RG54608); Newlife-The Charity for Disabled Children (Grant Number 07/20); the World Cancer Research Fund International (Grant Number 2004/03); and the National Institute for Health Research Cambridge Biomedical Research Centre. $\mathrm{KO}$ is supported by the Medical Research Council (Unit Programme number: MC_UU_12015/2). None of the funding bodies influenced the design of the study or the collection, analysis or the interpretation of the data used in this study. Neither did they influence the writing of this manuscript. The preprint of this manuscript is available (bioRxiv 556886; doi: https://doi.org/10.1101/556886).

Authors' contribution $\mathrm{CP}$ conceived and designed the analysis, evaluated some of the data and wrote the initial draft of the manuscript. BF analysed some of the data and commented on the initial draft of the manuscript. $\mathrm{KO}, \mathrm{IH}, \mathrm{CA}$ and DD designed, established and oversee the Cambridge Baby Growth Study. All authors commented on early drafts of the manuscript and approved the submission of the final draft.

\section{Compliance with ethical standards}

Conflict of interest The authors declare that they have no conflict of interest.

Statement of human and animal rights All procedures followed were in accordance with the ethical standards of the responsible committee on human experimentation (institutional and national)and with the Helsinki Declaration of 1975, as revised in 2008.

Informed Consent Informed consent was obtained from all patients for being included in the study.

Open Access This article is distributed under the terms of the Creative Commons Attribution 4.0 International License (http://creativeco mmons.org/licenses/by/4.0/), which permits unrestricted use, distribution, and reproduction in any medium, provided you give appropriate credit to the original author(s) and the source, provide a link to the Creative Commons license, and indicate if changes were made.

\section{References}

1. American Diabetes Association (2010) Diagnosis and classification of diabetes mellitus. Diabetes Care 33(Supplement 1):S62-S69

2. American Diabetes Association (2018) Classification and diagnosis of diabetes: standards of medical care in diabetes-2018. Diabetes Care 41(Supplement 1):S13-S27

3. Chu SY, Callaghan WM, Kim SY et al (2007) Maternal obesity and risk of gestational diabetes mellitus. Diabetes Care 30:2070-2076 
4. Petry CJ (2010) Gestational diabetes: risk factors and recent advances in its genetics and treatment. Br J Nutr 104:775-787

5. Salzer L, Yogev Y (2014) Complications of gestational diabetes. In: Petry CJ (ed) Gestational diabetes: origins, complications and treatment. CRC Press, Boca Raton, pp 95-115

6. Damm P, Houshmand-Oeregaard A, Kelstrup L, Lauenborg J, Mathiesen ER, Clausen TD (2016) Gestational diabetes mellitus and long-term consequences for mother and offspring: a view from Denmark. Diabetologia 59:1396-1399

7. Zhu Y, Zhang C (2016) Prevalence of gestational diabetes and risk of progression to type 2 diabetes: a global perspective. Curr Diab Rep 16:7

8. Mitchell S, Shaw D (2015) The worldwide epidemic of female obesity. Best Pract Res Clin Obstet Gynaecol 29:289-299

9. An R, Ji M, Zhang S (2018) Global warming and obesity: a systematic review. Obes Rev 19:150-163

10. Schmidt MI, Matos MC, Branchtein L et al (1994) Variation in glucose tolerance with ambient temperature. Lancet 344:1054-1055

11. Booth GL, Luo J, Park AL, Feig DS, Moineddin R, Ray JG (2017) Influence of environmental temperature on risk of gestational diabetes. CMAJ 189:E682-E689

12. Retnakaran R, Ye C, Kramer CK et al (2018) Impact of daily incremental change in environmental temperature on beta cell function and the risk of gestational diabetes in pregnant women. Diabetologia 61:2633-2642

13. Katsarou A, Claesson R, Ignell C, Shaat N, Berntorp K (2016) Seasonal pattern in the diagnosis of gestational diabetes mellitus in southern Sweden. J Diabetes Res 2016:8905474

14. Moses RG, Wong VC, Lambert K, Morris GJ, San Gil F (2016) Seasonal changes in the prevalence of gestational diabetes mellitus. Diabetes Care 39:1218-1221

15. Verburg PE, Tucker G, Scheil W, Erwich JJ, Dekker GA, Roberts CT (2016) Seasonality of gestational diabetes mellitus: a South Australian population study. BMJ Open Diabetes Res Care 4:e000286

16. Chiefari E, Pastore I, Puccio L et al (2017) Impact of seasonality on gestational diabetes mellitus. Endocr Metab Immune Disord Drug Targets 17:246-252

17. Vasileiou V, Kyratzoglou E, Paschou SA, Kyprianou M, Anastasiou E (2018) The impact of environmental temperature on the diagnosis of gestational diabetes mellitus. Eur J Endocrinol 178:209-214

18. Moses R, Griffiths R (1995) Is there a seasonal variation in the incidence of gestational diabetes? Diabet Med 12:563-565

19. Janghorbani M, Stenhouse E, Jones RB, Millward A (2006) Gestational diabetes mellitus in Plymouth, U.K.: prevalence, seasonal variation and associated factors. J Reprod Med 51:128-134

20. Ferrara A (2007) Increasing prevalence of gestational diabetes mellitus: a public health perspective. Diabetes Care 30(Suppl 2):S141-S146

21. Feig DS, Hwee J, Shah BR, Booth GL, Bierman AS, Lipscombe LL (2014) Trends in incidence of diabetes in pregnancy and serious perinatal outcomes: a large, population-based study in Ontario, Canada, 1996-2010. Diabetes Care 37:1590-1596

22. Khalifeh A, Breathnach F, Coulter-Smith S, Robson M, Fitzpatrick C, Malone F (2014) Changing trends in diabetes mellitus in pregnancy. J Obstet Gynaecol 34:135-137

23. Sella T, Shalev V, Elchalal U, Chovel-Sella A, Chodick G (2013) Screening for gestational diabetes in the 21 st century: a population-based cohort study in Israel. J Matern Fetal Neonatal Med 26:412-416

24. Wang Y, Chen L, Xiao K et al (2012) Increasing incidence of gestational diabetes mellitus in Louisiana, 1997-2009. J Womens Health (Larchmt) 21:319-325
25. Huy C, Loerbroks A, Hornemann A, Röhrig S, Schneider S (2012) Prevalence, trend and determining factors of gestational diabetes in Germany. Geburtshilfe Frauenheilkd 72:311-315

26. Prentice P, Acerini CL, Eleftheriou A, Hughes IA, Ong KK, Dunger DB (2016) Cohort profile: the Cambridge Baby Growth Study (CBGS). Int J Epidemiol 45:35

27. Petry CJ, Ong KK, Hughes IA, Acerini CL, Frystyk J, Dunger DB (2017) Early pregnancy-associated plasma protein A concentrations are associated with third trimester insulin sensitivity. J Clin Endocrinol Metab 102:2000-2008

28. National Collaborating Centre for Women's and Children's Health (2015) Diabetes in pregnancy: management of diabetes and its complications from preconception to the postnatal period. RCOG Press, London

29. International Association of Diabetes and Pregnancy Study Groups Consensus Panel, Metzger BE, Gabbe SG, Persson B et al (2010) International Association of Diabetes and Pregnancy Study Groups recommendations on the diagnosis and classification of hyperglycemia in pregnancy. Diabetes Care 33:676-682

30. Levy JC, Matthews DR, Hermans MP (1998) Correct homeostasis model assessment (HOMA) evaluation uses the computer program. Diabetes Care 21:2191-2192

31. Lauzon-Guillain B, Wijndaele K, Clark M et al (2012) Breastfeeding and infant temperament at age three months. PLoS ONE 7:e29326

32. Manley SE, Stratton IM, Clark PM, Luzio SD (2007) Comparison of 11 human insulin assays: implications for clinical investigation and research. Clin Chem 53:922-932

33. Petry CJ, Ong KK, Dunger DB (2018) Age at menarche and the future risk of gestational diabetes: a systematic review and dose response meta-analysis. Acta Diabetol 55:1209-1219

34. Petry CJ, Ong KK, Hughes IA, Acerini CL, Dunger DB (2018) The association between age at menarche and later risk of gestational diabetes is mediated by insulin resistance. Acta Diabetol 55:853-859

35. Haggarty P, Campbell DM, Duthie $S$ et al (2009) Diet and deprivation in pregnancy. Br J Nutr 102:1487-1497

36. Laraia B, Messer L, Evenson K, Kaufman JS (2007) Neighborhood factors associated with physical activity and adequacy of weight gain during pregnancy. J Urban Health 84:793-806

37. Collier A, Abraham EC, Armstrong J, Godwin J, Monteath K, Lindsay R (2017) Reported prevalence of gestational diabetes in Scotland: the relationship with obesity, age, socioeconomic status, smoking and macrosomia, and how many are we missing? J Diabetes Investig 8:161-167

38. Cullinan J, Gillespie P, Owens L, Avalos G, Dunne FP, ATLANTIC DIP collaborators (2012) Is there a socioeconomic gradient in the prevalence of gestational diabetes mellitus? Ir Med J 105(5 Suppl):21-23

39. Janghorbani M, Stenhouse EA, Jones RB, Millward BA (2006) Is neighbourhood deprivation a risk factor for gestational diabetes mellitus? Diabet Med 23:313-317

40. Huvinen E, Eriksson JG, Stach-Lempinen B, Tiitinen A, Koivusalo SB (2018) Heterogeneity of gestational diabetes (GDM) and challenges in developing a GDM risk score. Acta Diabetol 55:1251-1259

41. Lavery JA, Friedman AM, Keyes KM, Wright JD, Ananth CV (2017) Gestational diabetes in the United States: temporal changes in prevalence rates between 1979 and 2010. BJOG 124:804-813

42. Tura A, Kautzky-Willer A, Pacini G (2006) Insulinogenic indices from insulin and C-peptide: comparison of beta-cell function from OGTT and IVGTT. Diabetes Res Clin Pract 72:298-301

Publisher's Note Springer Nature remains neutral with regard to jurisdictional claims in published maps and institutional affiliations. 